\title{
Unusual association between spinal cord tumour and perioperative arrhythmia
}

\author{
Vikas Chauhan, Ashish Bindra, Parmod K. Bithal
}

\begin{abstract}
There are multiple causes of perioperative arrhythmias. Some have underlying cardiac disease while others accompany systemic pathology. Use of anaesthetic agents in the intraoperative period is also a known cause of rhythm abnormalities. Preoperative benign arrhythmias may progress to serious ones in intraoperative period. The trigger may be a transient insult such as hypoxemia, cardiac ischaemia, catecholamine excess or electrolyte abnormality. Thus, presence of arrthymia in the preoperative period adds to preoperative work-up and especially in the elective surgery settings, they call for additional opinion and patient evaluation. However, not all arryhthmias are amenable to drug treatment and modalities like pacing, some require just careful watch in the perioperative period. We report a patient with thoracic intramedullary space occupying lesion who presented to us with multiple ventricular ectopics on electrocardiography, which eventually disappeared with tumour removal.The case highlights the association of multiple ectopics with spinal tumour and their management.
\end{abstract}

Key words: Aetiology, anaesthesia, arrhythmia, intramedullary tumour, perioperative

\section{INTRODUCTION}

Perioperative arrhythmias are not uncommon trouble makers. Diagnosis on electrocardiography (ECG), adds to preoperative work-up and in the elective surgery settings, they call for additional opinion and patient evaluation. Arrhythmias are multifactorial and many have underlying cardiac disease but some may accompany systemic pathology.$^{[1]}$ Likewise, some are amenable to drug treatment and modalities like pacing, others require just careful watch in the perioperative period. ${ }^{[2]}$ We report a case of a patient with thoracic intra-medullary space occupying lesion (IMSOL) who presented to us with multiple ventricular ectopics (VPB) on ECG, which disappeared with tumour removal.

\begin{tabular}{|l|l|}
\hline \multicolumn{2}{|c|}{ Access this article online } \\
\hline Quick Response Code: & Website: \\
\hline & www.jnaccjournal.org \\
\hline & \\
\hline & DOI: \\
\hline & $10.4103 / 2348-0548.154237$ \\
\hline
\end{tabular}

\section{CASE REPORT}

A 39-year-old female patient with chief complaints of backache since 1 year and paraesthesia and weakness of lower limbs since 3 months was posted for surgery for removal of $2^{\text {nd }}-6^{\text {th }}$ thoracic IMSOL. Presumptive diagnosis on magnetic resonance imaging (MRI) was ependymoma. There were frequent VPBs (4-5 every 10 beats) in ECG [Figure 1]. Due to weakness of lower limbs, patient was not able carry out her daily activities very well. Her breath holding time was 20 seconds. There was no other significant history of medical illness. Blood investigations including routine haematology, serum biochemistry and $X$-ray chest were within normal limits. The preoperative haemoglobin was $12 \mathrm{~g} / \mathrm{dl}$, serum sodium, potassium was $140 \mathrm{mEq} / 1$ and $3.7 \mathrm{mEq} / 1$, respectively. In view of multiple VPBs and intraoperative risk of increase in arrhythmias with anaesthesia and tumour manipulation, cardiologist evaluation was done. According to the cardiologist, multiple VPCs of right ventricular outflow origin with long coupling interval were present on ECG, which were likely to be benign in presence of good effort tolerance and normal chest X-ray. No further evaluation was required and the patient was taken for laminectomy and removal of IMSOL under general anaesthesia. In the

Department of Neuroanesthesiology, All India Institute of Medical Sciences, New Delhi, India

Address for correspondence:

Dr. Ashish Bindra, Department of Neuroanaesthesiology, All India Institute of Medical Sciences, New Delhi - 110 029, India.

E-mail: dr_ashi2208@yahoo.com 


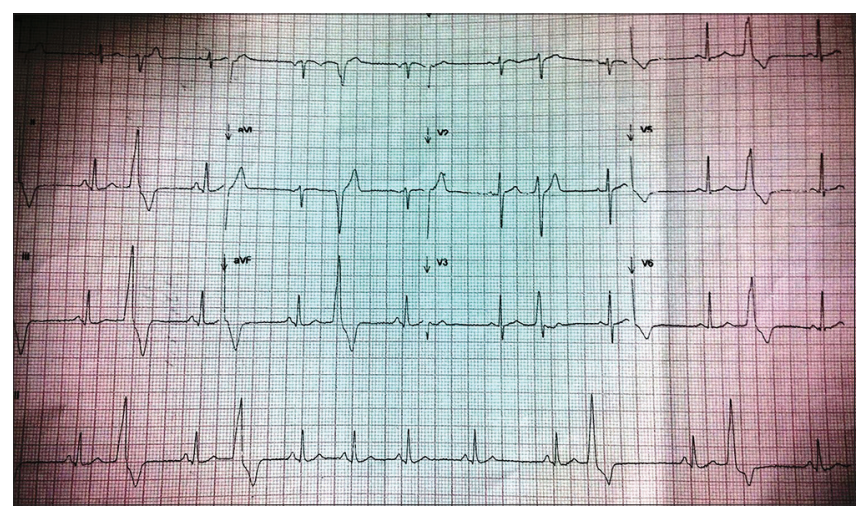

Figure 1: Preoperative electrocardiography (ECG) showing multiple ventricular premature beats

operation room, her baseline pulse rate was 83 beats per minute, blood pressure was $124 / 76 \mathrm{~mm}$ of $\mathrm{Hg}$ and serum electrolytes were - sodium $138 \mathrm{mEq} / 1$ and potassium 3.7 $\mathrm{mEq} / 1$ on arterial blood gas analysis. An intraoperative 5 lead ECG was attached to the patient, which still revealed the same findings. To keep a watch on haemodynamic status, preinduction invasive blood pressure monitoring was used apart from standard non-invasive monitoring. Anaesthesia was induced using $2 \mathrm{mcg} / \mathrm{kg}$ fentanyl, $3 \mathrm{mg} / \mathrm{kg}$ propofol and $1 \mathrm{mg} / \mathrm{kg}$ rocuronium. Sevoflurane in mixture of $40 \%$ oxygen and $60 \%$ nitrous oxide was used for maintenance. Fentanyl and vecuronium bolus were given as and when required. Normothermia and normocapnia was maintained throughout the procedure. Hourly blood gas analysis was done which ensured normal blood gas parameters. During surgery ECG remained unchanged from the preoperative pattern. There was no haemodynamic instability. By the time tumour resection was complete, ectopics reduced significantly. Surgery was completed uneventfully and patient was extubated. In the postoperative period, no arrhythmias were seen on the monitor [Figure 2]. On the second postoperative day, a 12 lead ECG was obtained which was within normal limits. Patient was followed till $7^{\text {th }}$ postoperative day during which period she remained free of arrhythmias and was discharged from hospital.

\section{DISCUSSION}

We report a case of patient with thoracic IMSOL having multiple preoperative VPBs, which gradually decreased and finally vanished following tumour excision. Occurrence of multiple ectopics in pre and intraoperative period and their subsequent disappearance following tumour resection highlight their benign nature and possible association with tumour.

Per se there is a high incidence of arrhythmias during anaesthesia. ${ }^{[3]}$ Preoperative benign arrhythmias may progress to serious ones in intraoperative period. The trigger may be a transient insult such as hypoxemia,

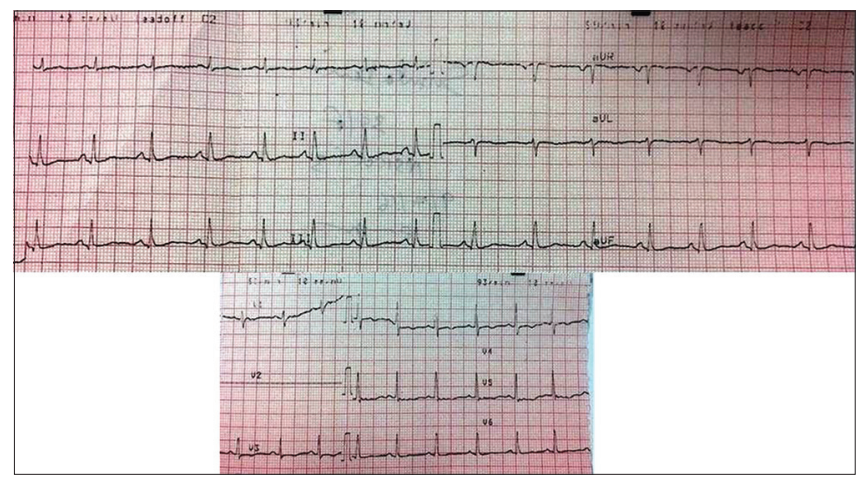

Figure 2: Postoperative normal electrocardiography (ECG)

cardiac ischaemia, catecholamine excess or electrolyte abnormality.

Volatile anaesthetics, including halothane, enflurane, sevoflurane and desflurane are known to produce arrhythmias. Halothane sensitizes the myocardium to endogenous and exogenous catecholamines. Sevoflurane may cause severe bradycardia and nodal rhythm when used in high concentrations during induction in infants while desflurane is known to prolong corrected QT interval (QTc) within the first minute of anaesthesia. Ketamine can facilitate the development of epinephrine-induced arrhythmias due to blocking of reuptake of norepinephrine. Other factors like electrolyte and blood gas abnormalities, drug interactions, brainstem stimulation, changes in autonomic nervous system tone and trauma to the heart are known to precipitate arrhythmias in perioperative period. Excessive hyperventilation, especially with low serum potassium levels, may lead to severe cardiac arrhythmias. ${ }^{[2,4]}$

VPBs account for $15 \%$ of observed arrhythmias under anaesthesia. ${ }^{[4]}$ They are much more common in anaesthetized patients with pre-existing cardiac disease. The treatment is to correct serum electrolyte and blood gas abnormalities. If the arrhythmia associated with haemodynamic instability, lidocaine $(1.5 \mathrm{mg} / \mathrm{kg})$ bolus can be given. Recurrent VPBs can be treated with a lidocaine infusion at $1-4 \mathrm{mg} / \mathrm{min}$; additional therapy includes judicious use of appropriate anti arrythmic medications or overdrive pacing.

Predominant parasympathetic activity resulting in profound bradycardia, arrythmias and cardiac arrest are well documented in patients with cervical and thoracic spinal cord injury. ${ }^{[5]}$ The mechanism of these arrhythmias appears to be related to changes in autonomic nervous system tone. The parasympathetic supply via the vagus is spared in most cases of spinal cord injury. Sympathetic outflow occurs below the T6 level and injuries above this level generally result in loss of input to sympathetic neurons. So, this autonomic 
imbalance occurring in injuries at or above T6 result in significant alterations in cardiovascular system control. ${ }^{[6]}$

Thus, high thoracic injuries cause cardiovascular complications due to loss of supra spinal control of sympathetic nervous system. ${ }^{[7]}$ There is scant literature regarding occurrence of cardiac rhythm abnormalities in patients with spinal cord tumours but similar mechanism may be held responsible for arrhythmias in these patients. VPBs have been reported in thoracic spine surgery intraoperatively due to surgical stimulation also. In rare instances, intense stimulation has led to asystole in these patients. ${ }^{[8,9]}$

In our case, the rhythm disturbances were present in the preoperative period and continued until tumour excision. We presume that IMSOL was potential source of arrhythmic activity in this patient, which got abolished with its surgical removal. Their occurrence in such a setting should not be a reason for delay or cancellation of an elective surgery. However, presence of preoperative arrhythmias likely due to spinal pathology should warn the physician and patient should be carefully monitored for worsening of arrhythmias and haemodynamic instability during tumour dissection. The predisposing factors should be kept in mind and drugs causing arrhythmias should be avoided. Further reporting is required to establish a relationship between preoperative rhythm disturbances and outcome in such patients.

\section{REFERENCES}

1. Thompson A, Balser JR. Perioperative cardiac arrhythmias. $\mathrm{Br}$ J Anaesth 2004;93:86-94.

2. Fisher DM. Preoperative cardiac dysrhythmias; Diagnosis and management. Anesthesiology 1997;86:1397-424.

3. Urwyler A, Scheidegger D. Arrhythmias and anaesthesia. Baillière's Clin Anaesthesiol 1993;7:2281-97.

4. Dua N, Kumra VP. Management of perioperative arrhythmias. Indian J Anaesth 2007;51:310-23.

5. Hector SM, Biering-Sørensen T, Krassioukov A, Biering-Sorensen F. Cardiac arrhythmias associated with spinal cord injury. J Spinal Cord Med 2013;36:591-9.

6. Julio C, Furlan JC, Fehlings MG. Cardiovascular complications after acute spinal cord injury: Pathophysiology, diagnosis, and management. Neurosurg Focus 2008;25:1-15.

7. Katz RL, Bigger JT Jr. Cardiac arrhythmias during anesthesia and operation. Anesthesiology 1970;33:193-213.

8. Rayner PR. Cardiac arrhythmias during high spinal surgery. $\mathrm{Br}$ Med J (Clin Res Ed) 1983;287:182.

9. Garstang SV, Miller-Smith SA. Autonomic nervous system dysfunction after spinal cord injury. Phys Med Rehabil Clin N Am 2007;18:275-96.

How to cite this article: Chauhan V, Bindra A, Bithal PK. Unusual association between spinal cord tumour and perioperative arrhythmia. J Neuroanaesthesiol Crit Care 2015;2:127-9.

Source of Support: Nil, Conflict of Interest: None declared. 